Sección Especial 


\title{
EL RETO EDUCATIVO UNIVERSITARIO
}

\author{
Iván Solano Zúñiga
}

Recibido 22-XI-2001

Resumen: Los estudiantes estamos en la universidad para especializarnos y aprender a ser personas. Este camino para ser personas perdura toda la vida, buscando una perfección que no podemos alcanzar, pero buscada con el afán de ser mejores cada día. Para esto, debemos comprometernos con nosotros mismos y con los demás; en este compromiso el educador está nuestro lado.

Necesitamos una serie de principios y valores que rijan nuestro diario acontecer; que conformen nuestra manera de ser.

En la universidad debemos enfrentar un tipo de educación diferente a la del colegio. Necesitamos hacernos dueños de la materia, y "exprimir" al profesor; el que debe predicar con el ejemplo para que nos unamos a la causa de aprender. Debemos darnos cuenta de que no podemos alcanzar nuestro objetivo siendo estudiantes mediocres.

Se requiere que pongamos el "todo" de nosotros; aceptando habilidades y debilidades, buscando siempre llegar a ser la "persona ideal".

En la educación hay que mirar cada momento con un ojo crítico. No debemos pensar en graduarnos y convertirnos en máquinas de trabajo; tratemos de ser personas; la educación y el empuje de la universidad nos dan la posibilidad de lograr algo que no muchos alcanzan.
- Aceptado 18-III-2002

Cerca del colegio San Luis Gonzaga en Cartago había un graffiti que decía así: "Si estudiar da frutos que estudien los árboles". Todos los días, después de lecciones, pasaba por ahí y me reía del mensaje, sin darme cuenta que esto no debía causar risa, sino preocupación por la ignorancia y la falta de conciencia de la persona que estampó su firma -un poco descriptiva- en dicha pared.

Ahora, con la oportunidad que gozo al recibir educación universitaria, me doy cuenta de que en realidad se puede hacer una reflexión sobre dicha frase. Los árboles dan frutos, igual que el estudio. Pero los árboles no dan frutos en cierto momento, ni por ciertos períodos, sino que lo hacen permanentemente, luego del momento en que están posibilitados para hacerlo. Así debemos ser nosotros, como los árboles, dar fruto, a lo largo de toda nuestra vida, cultivarnos siempre, y no dejar estancada nuestra cosecha.

¿Cómo cosechamos?, es más, ¿cómo sembramos? La respuesta es simple a primera vista, pero profunda si queremos interesarnos realmente en algo que nos atañe a todos los seres humanos. La educación; sí, eso que desde chiquitos nos tratan de inculcar y que nunca llegamos a perfeccionar. Ese es el medio para cosechar frutos, y no cualquier fruto, sino buenos frutos, frutos que nos sirvan a nosotros, a los que nos rodean y a los que nos siguen. 
Así como yo, hay muchos jóvenes que se encuentran en un punto clave en su vida, tienen en sus manos la oportunidad única de recibir educación universitaria, un paso en que tal vez no lleguemos a ser personas, pero en el que aprenderemos cómo llegar a ser tales. Recibimos una educación adecuada, pero no sabemos cómo afrontarla ni aprovecharla, dada nuestra ignorancia sobre lo que podemos alcanzar poniendo todas nuestras fuerzas en esta etapa de la vida.

En el siguiente ensayo, profundizo en la persona, el centro de la educación, y cómo debe enfrentarse la educación desde el punto de vista del estudiante universitario, el joven, así como yo, de 19, 20 ó 21 años, que empieza a darse cuenta del camino que debe seguir en la vida, y que desea saber cómo seguirlo mediante una adecuada educación. Para el escrito me basaré en las ideas de José Luis González-Simancas y su libro: Educación: Libertad y compromiso, y de Guillermo Molina Guzmán en Crisis de la educación costarricense, e ideas propias que nacen de mi experiencia como estudiante.

\section{Educación}

En este punto no voy a entrar en definiciones, ya que cualquier definición que pueda yo dar no aportaría nada al ya tan sonado tema de la educación, al contrario, voy a tocar algunos puntos importantes sobre educación que nos deben interesar a todos, no sólo al estudiante universitario.

Primero, debemos darnos cuenta de que la educación no es algo que podemos dejar botado; ésta implica un proceso que se lleva a lo largo de toda la vida; en primera instancia, porque el hombre es un ser imperfecto por naturaleza, y la educación busca un perfeccionamiento de la persona, cosa que nunca se va a conseguir, pero que se puede seguir buscando con la idea de ser mejor cada día. Y, en segunda instancia, porque la humanidad avanza muy rápido en cuanto a tecnología, literatura y avances científicos, y no podemos quedarnos atrás en un mundo que cambia constantemente. Así queda claro, que no debemos creer que ya, porque estamos en las últimas etapas de nuestra carrera, o porque ya tenemos edad suficiente, debamos detener nuestra acción de educarnos.

Pero no podemos educarnos, sea cual sea la manera en que enfrentemos este reto, si no tenemos bien definido un compromiso con nosotros y con los demás para educarnos y sacar lo mejor de nosotros. En este compromiso debemos ser disciplinados y constantes; si no, nos quedaremos en la mediocridad de muchos que tienen la oportunidad de ser mejores, y se rehúsan a dar un esfuerzo total. Debemos ser valientes y ser fuertes, para hacerle frente a situaciones y dificultades que forjen nuestro carácter, que le den fuerza a nuestra integridad, integridad que nos ayudará a ser personas.

Debemos entender que este compromiso no es algo que afrontamos solos. El educador adquiere este compromiso a nuestro lado. Nosotros aprendemos de él, y él de nosotros; los dos estamos enfrascados en ese "círculo vicioso" que llamamos educación, donde cada uno pone su aporte para aprender; unos, para aprender a aprender; y otros, para aprender de los que aprenden.

Para comenzar, deberíamos regir nuestra vida por una serie de principios y valores, que reflexionando un poco, son semejantes; como lo hace notar José Luis González-Simancas. Sin darse cuenta, este autor nos presenta estos dos términos de una manera semejante cuando dice: "las ideas se transforman en principios desde el mismo momento en que las hacemos nuestras, hasta tal punto que llegan a orientar nuestra actuación como personas y como educadores" (GonzálezSimancas, 1992, p. 40), y luego nos dice de los valores: 
"La tan traída y llevada educación en los valores supone, en efecto, todo un proceso de asunción de los valores: lo que quiere decir que hay que ir haciéndolos propios progresivamente y mediante una implicación personal que es la propia del compromiso." (González-Simancas, 1992, p. 80).

por lo que queda demostrado que ambos debemos hacerlos nuestros, y luego de eso, aprovecharlos de la manera que nos sea correcta para regir nuestro diario acontecer.

Pero todavía no entramos en el estudiante universitario, el que debe ser y el que tal vez realmente es, cosa que haremos en los siguientes apartados. Pero, antes de entrar en el estudiante y cómo debe enfrentar el reto educativo universitario, quiero mostrar la perspectiva sobre el cambio que se sufre del colegio a la universidad.

\section{El cambio del cole a la $U$}

Aquí creo que muchos asienten conmigo cuando digo que este cambio es un poco difícil, y no porque la mayoría de estudiantes sean malos estudiantes, sino porque el alumno viene acostumbrado a un tipo de educación totalmente distinto.

En el colegio, el estudiante está acostumbrado a no pensar ni analizar, a responder, a memorizar y a hacer suyos pensamientos que le son introducidos como ley. No hay un espacio de discusión, para que éste se desarrolle y tenga un juicio crítico. Por esto es que nos cuesta, a veces a los estudiantes universitarios, responder a las necesidades que presentan los cursos en que se nos da espacio para revelar nuestros puntos de vista. Además, aunque tengamos puntos de vista encontrados hacia algunas ideas, es casi seguro que la mayoría de los estudiantes no está acostumbrada a hablar en público y a exponer con seguridad sus ideas, por lo que muchas veces teme exponerse de esta manera.

Al estudiante colegial no se le da una lugar para que se exprese, no sólo intelectualmente, sino culturalmente; se le impulsa en el campo deportivo, pero no se le enseña que puede enunciar, formular y proclamar sus ideas, canciones, poemas, y toda su producción creativa, por lo que a la universidad llega un estudiante que no puede responder a algo que nunca ha conocido, como lo es la expresión en todo su sentido.

Por otro lado, el estudiante se enfrenta ahora a un tipo de responsabilidad totalmente diferente; los trabajos son más serios y más profundos. Hay que tomar en cuenta que, además del contenido, el formato del trabajo es muy diverso. El alumno encara ahora citas bibliográficas, bibliografías, marcos teóricos y otro conjunto de teoría sobre investigación muy diferente a lo que vivía en el colegio, donde sus trabajos sólo consistían en introducción, desarrollo y conclusión. Los profesores dan la ayuda de guiar al estudiante en cuanto a los errores de formato y de fondo; algo positivo, dado que en el colegio el estudiante realiza investigaciones, pero pocas veces se da cuenta de los errores en que incurrió. En la universidad, el estudiante realmente aprende de sus errores.

El universitario debe dedicar más tiempo al estudio, ya no basta con estudiar unos días antes del examen como solía hacerlo; debe estar siempre atento a lo que explique el profesor, y no esperar que la materia más fácil es la que aparece en la prueba, como sucedía mucho en el colegio. Los exámenes requieren de un estudio profundo; la dificultad de los ejercicios exige un nivel mayor de entendimiento, un nivel donde el estudiante sea dueño de la materia. Por esto, el período universitario es un momento en el cual no se deben dejar las dudas a un lado; por el contrario, debe "socavarse" al profesor hasta llegar al entendimiento, cosa que no hace el estudiante regular en el colegio. Para esta situación, existen los grupos de estudio y las horas de consulta, que son de gran provecho e importancia, una medida muy inteligente, pero que lastimosamente no es aprovechada por la mayoría de los estudiantes. 


\section{El estudiante utópico - la persona}

Para entrar a esta parte debemos de nombrar un punto que puede afectar el desempeño de los estudiantes universitarios. Aparte de todas las dificultades que representa el salto del colegio a la universidad, algunos jóvenes sufren de una "adolescencia prolongada", citando a Gerardo Castillo: "Son jóvenes por su edad cronológica, pero no por su madurez, la juventud es para ellos solamente una adolescencia prolongada" (González-Simancas, 1992, p. 79); esa inmadurez puede hacer ver la educación desde un ángulo distinto.

Pero, para poder vencer el reto universitario hay que dar un primer paso; debemos poner el "todo" de nosotros; tenemos que aprender a llegar a ser; a dar el cien por ciento que hay dentro de nosotros; rendir todo lo que tenemos; conocernos, aceptando nuestras habilidades y debilidades, reconociendo estos hechos sin sentirnos superiores por nuestros bienes, ni inferiores por las limitaciones que tengamos, para comenzar el camino para llegar a ser esa persona completa que buscamos. $\mathrm{Y}$ todo este pensamiento debe ir en busca de llegar a ser la persona ideal, aquella que ha crecido culturalmente, que pone todo en su trabajo, su estudio, su familia y sus lazos afectivos; la persona que tiene buenos sentimientos y tiene una disposición para con los demás de servicio, persona que busca su bien y el de los otros, dejando de lado el egoísmo.

¿Y cómo acercarnos al nivel de esa persona ideal? Bueno, para llegar allí hay un largo camino que podemos recorrer y que incluye la educación como base. En primer lugar, debemos llenarnos de hábitos intelectuales y morales; éstos facilitan la acción del que aprende, "ejercitando la inteligencia y la voluntad aplicadas a actividades concretas" (González-Simancas, 1992 , p. 217). Hay que acercarse al punto de tener una curiosidad intelectual, que nos induzca a informarnos, a leer, y a vivir, en síntesis a llegar a eso que llama González-Simancas el "buen intelectual"; el que "no tiene por qué ser una persona que exclusivamente se dedique a cultivar su inteligencia y los conocimientos de su especialidad" (González-Simancas, 1992, p. 71), al que "si no se manifiesta en su vida como una persona humana, es decir, sociable, atenta a los demás, abierta y disponible, a su persona, y a su vida le faltarán algunos matices importantes" (González-Simancas, 1992, p. 71).

La universidad trata de formar personas íntegras, personas con temple, que sepan darle cara a las situaciones que se le enfrentan. Una manera de hacer conocer estas situaciones es con la carga de materias, la responsabilidad, la investigación de temas diversos, y el estudio bajo presión; ya lo dijo González-Simancas "ese tipo de contrariedades que surgen en la vida diaria -cuando decimos que la vida se hace «dura»- son otras tantas circunstancias sociales que forjan a las personas íntegras, haciéndolas enteras" (González-Simancas, 1992, p. 89), por lo que el estudiante llega a familiarizarse con el hecho de hallarse en situaciones difíciles y darles una respuesta adecuada. Si aprende a solucionar las exigencias que presenta el nivel académico de la universidad, con facilidad logrará luego responder a las dificultades de la vida, razonando de una buena manera. El estudiante que llega a responder adecuadamente a estas exigencias, aprende a estar bajo presión, pero debe aprender también a aprovechar su tiempo libre, su tiempo de ocio:

\footnotetext{
"Estos momentos que le quedan para sí mismo deben ser explotados; su valor es importante, ya sea para reflexionar, compartir con los demás, para hacer un pequeño alto al esfuerzo que comúnmente se realiza, para hacer alguna actividad entretenida y enriquecedora" (González-Simancas, 1992, p. 132).
}

El universitario no debería dejar pasar estos momentos conectado al televisor, llenando su cerebro de basura en la mayoría 
de las veces, o cargando de violencia su cerebro con la máquina de videojuegos.

$\mathrm{Y}$ por último, un punto que no puede faltar, y que es esencial para el alcance de nuestro objetivo: la disciplina. Estamos acostumbrados a que ésta última nos la impongan siempre; pero es un hábito que debemos hacer nuestro, para llegar a la autodisciplina; que "nace de la voluntad de los componentes del grupo, que obedecen a la exigencia de orden por propia decisión y aceptación de la conveniencia de ese orden, como condición para conseguir lo que se proponen o se les propone" (González-Simancas, 1992, p. 196). Esta autodisciplina es la guía que nos acerca al fin de nuestro compromiso. Es la que nos hace estudiar solos; investigar, si tenemos dudas; ir un paso adelante del profesor si es que se puede. Sólo la disciplina, luego de haber encontrado nuestro camino, nos facilitará la llegada al objetivo de nuestra educación.

\section{El educador}

Como lo demuestra González-Simancas en su libro, la educación no se centra sólo en el educando, sino que también abarca el papel del educador, el que va de la mano con el anterior en una retroalimentación, a lo largo de todo el viaje de la educación. Por esto, en esta parte hago referencia a algunos puntos sobre el educador, su influencia y algunas características.

Como antes nombré, la educación es un proceso que no termina a lo largo de la vida, por lo que el educador no es el dueño del saber sino que es, a la vez, un educando.

Uno de los medios más importantes para la transmisión del conocimiento es la comunicación, lazo que une al educando y al educador. El educador debe ser alguien que sepa comunicar los contenidos de su materia; de no ser así, o no poder comunicarlos, no debería estar en ese puesto de ninguna manera. Este es un problema que he visto presente en la universidad; me he encontrado con profesores que conocen muy bien la materia; pero eso no los hace capaces de transmitirla como debe ser; no cuentan con estudios de pedagogía, cosa que afecta en la mayoría de las veces al estudiante.

Otros aspectos que definen a un mal docente son: si su conducta es incongruente a lo que exige; es un desordenado que pide orden; exige interés y el mismo no lo demuestra; y así, otras actitudes. Este no logra que el estudiante se una a la causa de aprender, por lo que no sólo se afecta él. En este momento, el estudiante comienza a seguir un mandato, sin razonar ni aprender. González-Simancas para este caso nombra a Ortega y Gasset que propuso que "se obedece a un mandato, se es dócil a un ejemplo", donde podríamos añadir nuestro propio dicho para hacer un símil: "Se atrapan más moscas con una gota de dulce que con un barril de mostaza".

La educación no tiene el objetivo de hacer robots que memoricen y respondan. No, la idea es sacarles provecho a lo estudiantes; no es ver qué aprender, sino qué deducen de lo aprendido; por lo que no hay que confundir "formación con pura información” (González-Simancas, 1992, p. 221). Este es tal vez un error que se da, tanto en la educación universitaria, como en la primaria y la secundaria. Lo puede aclarar mejor la siguiente idea de González-Simancas:

"En el contexto de una enseñanza de calidad es absurdo y paradójico, por ejemplo, un profesor que se limite a «transmitir» un cúmulo de conocimientos a sus alumnos sin tratar de que encuentren el significado o el sentido que esos contenidos científicos tienen, no sólo para la ciencia, sino para la sociedad de los humanos y para los propios alumnos." (González-Simancas, 1992, p. 216).

Algo que incentiva al estudiante a buscar sus ideales es tener, en muchos casos como profesores en nuestra universidad, a personas que realmente aman lo que hacen, que luchan por un mundo mejor, y hasta profesores que son reconocidos 
escritores, doctores y especialistas, que nos demuestran que algún día podemos llegar ser como ellos y mejores. El estudiante se llena del espíritu de trabajo que tienen estas personas y desea seguir sus pasos, pero se da cuenta de que no puede llegar al nivel de estas personas siendo un estudiante mediocre, por lo que allí comienza el cambio para bien.

Sólo hace falta que el alumno se dé cuenta de en qué punto está fallando, dónde está él mismo impidiéndose su desarrollo como persona, para que comience a dar resultados, a estudiar más fuerte, y a buscar la utopía que hay en cada uno de nosotros, utopía que permito decir yo mismo he encontrado en mí, y pienso tratar de alcanzar. Todos los estudiantes deben llegar a ese punto donde encuentren un verdadero llamado a responder a la universidad, un punto de quiebra donde empiece la escalada hacia el éxito en todo el sentido de la palabra.

En conclusión, creo que es válido decir que la educación como fin para una persona requiere de un compromiso muy serio, al que debe responderse por el resto de la vida, donde hay que mirar cada segundo con un ojo crítico, para saber si estamos dando la talla en nuestra educación, si estamos fallando, o si algo externo nos hace fallar. $\mathrm{Y}$, donde esté en nuestras posibilidades, cambiar lo que nos impide realizarnos. El universitario promedio no aprovecha la oportunidad que tiene en sus manos, la de ser una persona íntegra, completa y plena. Se centra más en lo que se enseña que en cómo se enseña, que debiera ser el punto central; y no se esmera por educarse integralmente, y llegar a ser esa persona de la que hablábamos. Espera salir de la universidad y simplemente ser un profesional, un funcionario, casi una máquina, no una persona con buenos sentimientos, cultivada, educada y plena. Alguien de bien social, que le sirva al país.

$\mathrm{Al}$ menos yo, espero hacer ese compromiso; sé que no puedo alcanzar la perfección, pero la educación y el empuje que me da la universidad para emprender mi rumbo, me dan un chance de ser alguien; una persona, lo que no muchos llegan a alcanzar en toda su plenitud.

\section{Referencias bibliográficas}

González-Simancas, José Luis. Educación: libertad y compromiso. Pamplona: EUNSA S.A. 1992.

Molina Guzmán, Guillermo. Crisis de la educación costarricense. San José: Litografía e Imprenta LIL. 2000.

Iván Solano Zúñiga

Estudiante de la Escuela de Ciencias de la Computación e Informática de la Universidad de Costa Rica 
COLABORADORES

Flora Eugenia Salas Madriz: Obtuvo la Licenciatura en Filosofía en 1993 y la Maestría en Administración Universitaria en 1997, en la Universidad de Costa Rica. Es estudiante del posgrado en Filosofía también en la Universidad de Costa Rica. En marzo del 2002, obtuvo el grado de Bachiller en Psicología en la Universidad Centroamericana de Ciencias Sociales.

Es profesora en la Universidad de Costa Rica desde 1994, donde se ha desempeñado como docente en la Sede del Atlántico, en la Cátedra de Estudios Generales e impartiendo cursos en diversas carreras de Educación e Informática. También ha sido profesora de grado y de posgrado en la Escuela de Administración Educativa, y ha trabajado como docente en las escuelas de Estudios Generales y de Trabajo Social en la Sede Rodrigo Facio, y tiene otras publicaciones en la Revista de Educación de la Universidad de Costa Rica.

Randolf Aguirre Aguirre: Bachiller en Ciencias de la Educación con énfasis en I y II Ciclos de la Universidad Estatal a Distancia. Licenciado en Ciencias de la Educación con énfasis en Administración Educativa de la Universidad Estatal a Distancia. Máster en Ciencias de la Educación con énfasis en Administración Educativa de la Universidad de Costa Rica.

Experiencia laboral: Profesor de Historia de la Cultura e historia de América Latina en la Universidad Estatal a Distancia: periodo febrero - diciembre del 2000. Asistente del director del departamento de Enseñanza Especial de Ministerio de Educación Pública (Actualmente).

Sonia Carballo Vargas: Profesora de la Facultad de Educación de la Universidad de Costa Rica. Asumió durante cuatro años la Dirección de la Escuela de Formación Docente. Fue profesora de Psicología en instituciones de educación secundaria y es coordinadora de la carrera de "Enseñanza de la Psicología” en la Escuela de Formación Docente. Durante más de veinte años ha sido profesora del curso de Educación Sexual y realiza labores de acción social mediante el curso-taller "Expresión de la sexualidad e inteligencia emocional", que se ofrece a toda persona interesada. Dentro de sus últimos artículos publicados están "La lección: su creación"; "Intervenciones para ayudar a construir la inteligencia emocional"; "Retos de la maestra y del maestro del Siglo XXI".

Ronald Soto Calderón: Bachiller en Educación Especial de la Universidad de Costa Rica. Licenciado en Educación Primaria de la Universidad de Costa Rica. Magíster en Evaluación Educativa de la Universidad de Costa Rica. Magíster en Educación Especial (ICSE-Universidad Pontificia de Comillas, Madrid, España. Profesor de la Escuela de Orientación y Educación Especial de la Universidad de Costa Rica. Profesor de la División de Educación Básica del CIDE de la 
Universidad Nacional, Heredia. Además ha sido profesor en la Universidad Estatal a Distancia en la Carrera de Psicopedagogía. Profesor en el Sistema de Estudios de Posgrado (Maestría en Estudios Interdisciplinarios sobre discapacidad), amplia experiencia en docencia y asesoría en el campo de las adecuaciones curriculares, evaluación, autismo y déficit atencional, y en general sobre aspectos de discapacidad. Actualmente se desempeña también como Investigador del Instituto de Investigaciones para el Mejoramiento de la Educación Costarricense (IIMEC).

Allan Abarca Rodríguez: Investigador en temas de política pública educativa, ha realizado estudios de posgrado -maestría académica- en Políticas Públicas (énfasis cuantitativo) en FLACSO sede académica de México; es Licenciado en Ciencias Políticas (UCR) y egresado de la Licenciatura en Educación Ciudadana (UNED). Se desempeña como profesor y coordinador de los cursos de 'Políticas Públicas y Toma de Decisiones' tanto en la Escuela de Ciencias Políticas como en el Posgrado Centroamericano en Ciencias Políticas. Actualmente es el investigador principal de "La Deserción Estudiantil en la UCR: un estudio de su magnitud y causas", investigación que se ejecuta en el Centro de Evaluación Académica (CEA); y de "La política pública de elevar la calidad de la educación superior: un análisis del proceso de autoevaluación y de acreditación nacional", inscrito en la Escuela de Ciencias Políticas y en el Instituto de Investigaciones Sociales.

Ana Lucía Villarreal Montoya: Es Magister Scientiae en Sociología de la Universidad de Costa Rica, Licenciada y
Bachiller en Ciencias de la Comunicación Colectiva de la Universidad de Costa Rica (UCR). Ha escrito en diferentes revistas sobre temáticas de comunicación, educación, género. Es autora del texto Principios de Comunicación Rural, publicado por la Editorial de la Universidad Estatal a Distancia (UNED). Actualmente se desempeña como profesora Adjunta de la Escuela de Ciencias de la Comunicación de la UCR y profesora de la Escuela de Ciencias Exactas y Naturales de la UNED. Coordina el Programa de Investigación en Educación y Género (PIEG) del Instituto de Investigaciones para el Mejoramiento de la Educación Costarricense (IIMEC), de la Facultad de Educación de la UCR y es investigadora en dicho instituto.

Laura Mora Zúñiga: Licenciada y Bachiller en Ciencias de la Educación con énfasis en Orientación. Docente de la Escuela de Orientación y Educación Especial de la Universidad de Costa Rica e Investigadora del IIMEC-UCR, Orientadora en el Colegio de Gravilias.

Gabriela Muñoz Porras: Licenciada y Bachiller en Ciencias de la Educación con énfasis en Orientación. Docente de la Escuela de Orientación y Educación Especial de la Universidad de Costa Rica, Recinto Paraíso e Investigadora del IIMEC-UCR, Orientadora en el San Gabriel La Salle.

Cecilia Villarreal Montoya: Magíster Scientiae en Estudios de la Mujer. Licenciada y bachiller en Ciencias de la Educación con énfasis en Orientación. Docente de la Escuela de Orientación y Educación Especial en la Universidad de Costa Rica e Investigadora del IIMEC-UCR. 
Tania Elena Moreira Mora: En 1984 obtuvo el título de Bachiller en la Enseñanza del Español de la Universidad Nacional y en 1985 el de Bachiller en Literatura y Lingüística con énfasis en Español de la Universidad Nacional. A partir de este año hasta 1994 laboró como docente en la enseñanza del Español en varios colegios públicos de la Dirección Regional de Educación de San Carlos y de Limón.

En 1995 y 1996 se desempeñó como Asesora de Evaluación en la Dirección Regional de Educación de Limón y en 1996 se graduó en la Maestría en Evaluación Educativa de la Universidad de Costa Rica.

Desde 1999 labora como Asesora Nacional de Macroevaluación de la División de Control de Calidad y Macroevaluación del Sistema Educativo del Ministerio de Educación Pública y en el año 2000 se graduó como Licenciada en Literatura y Lingüística con énfasis en Español de la Universidad Nacional.

Jimmy Bolaños González: Es abogado y notario público, con una amplia trayectoria en la función pública, que incluye diez años como asesor jurídico de la Dirección General de Servicio Civil, instructor y conferencista, profesor universitario, miembro del Tribunal de Carrera Docente y del Tribunal de Árbitros Arbitradores del Colegio de Microbiólogos y Químicos Clínicos. Actualmente se desempeña como Jefe a.i. de la oficina Jurídica de la Universidad Estatal a Distancia. Tiene a su haber múltiples artículos en materia de empleo público y publicaciones como: La Reorganización de las Dependencia Públicas, Derecho Disciplinario de la Función Pública y Estatuto de Servicio Civil con comentarios, anotado y concordado.
María Elena Arce Urbina: Es Magíster Scientiae en Química. Profesora Adjunta de la Sede del Atlántico de la UCR, con amplia experiencia en la enseñanza de la Química Orgánica, Química Analítica y técnicas de trabajo en el laboratorio químico.

Coordinadora de diferentes proyectos de Extensión Docente en pro del mejoramiento en la enseñanza de las ciencias en la educación primaria y secundaria.

Autora del libro "Mis Primeros Experimentos" de la editorial de la UNED (2000).

También ha trabajado en diferentes proyectos de investigación en el campo de la Química, en donde ha realizado varias publicaciones.

Maureen Meneses Montero: Magister Scientae en Salud Pública por la Universidad de Costa Rica, Licenciada en la Enseñanza de la Educación Física. Profesora Catedrática de la Escuela de Educación Física y Deportes de la U.C.R. Coordinadora de Actividad Deportiva, profesora de cursos de natación, métodos de enseñanza en la educación física, psicomotricidad en la educación inicial. Ponente nacional e internacional en congresos, simposios y seminarios cuya temática central sea la psicomotricidad en el ciclo inicial y transición. También labora como profesora supervisora en las prácticas docentes y profesionales en la carrera de la enseñanza de la educación física. Miembro de la Comisión Nacional de Salud Escolar. Actualmente funge como subdirectora de la Escuela de Educación Física y Deportes.

\section{María de los Ángeles Monge Alvarado:} Egresada del la Maestría Académica en Administración Pública, Licenciada en la Enseñanza de la Educación 
Física, labora como docente (profesora asociada) en la Escuela de Educación Física y Deportes de la U.C.R. Imparte los cursos de desarrollo motor, psicomotricidad en la educación inicial, educación física adaptada, construcción de materiales didácticos, investigación cuantitativa, tecnología educativa y recursos didácticos, además es profesora de actividad deportiva. Miembro de la Comisión Nacional Movámonos Costa Rica, Miembro del Consejo Director del Programa de Salud Integral para los cantones de Montes de Oca, Curridabat y La Unión. Ponente nacional e internacional en congresos, simposios y seminarios cuya temática central sea la psicomotricidad y la educación física adaptada.

Jeanneth Cerdas Núñez: Es Máster en Planificación Curricular de la Universidad de Costa Rica, Bachiller en Educación Preescolar y Licenciada en Educación Preescolar de la Universidad de Costa Rica. Ha trabajado como Docente de Educación Primaria y como Docente de Educación Preescolar. También se ha desempeñado como supervisora de Práctica Docente de la carrera de Educación Preescolar de la Universidad de Costa Rica y como colaboradora de la Feria Nacional de Ciencia y Tecnología. Actualmente labora en el Ministerio de Educación Pública como Docente de Educación Preescolar en el Jardín de Niños Sarita Montealegre. También en la actualidad está colaborando como Supervisora de Práctica Docente en la carrera de Educación Preescolar de la Universidad de Costa Rica.

Ana Polanco Hernádez: Es Máster en Planificación Curricular de la Universidad de Costa Rica, Bachiller en Educación Preescolar y Licenciada en Educación Preescolar de la Universidad de Costa Rica. Trabajó como Docente de Educación Preescolar, durante 16 años, de los cuales los últimos 12 los laboró en la Sección Preescolar de la Escuela Nueva Laboratorio de la Universidad de Costa Rica. Desde 1986 y hasta la fecha se ha desempeñado como profesora de la Sección Preescolar de esta universidad, en cursos como Experiencia Profesional II (supervisora de Práctica Docente), Estudios Sociales en la Educación Inicial, Estrategias para el desarrollo de la creatividad del niño, Didáctica en la Educación Inicial I, colaboradora de la Feria Nacional de Ciencia y Tecnología, coordinadora del Proyecto denominado "Aulas de Prekínder en Instituciones Públicas de Educación Inicial", y directora de Seminarios de Graduación para optar al título de Licenciatura en Educación Preescolar.

Ana Patricia Rojas Núñez: Es Máster en Planificación Curricular de la Universidad de Costa Rica, Licenciada en Educación Preescolar de la Universidad de Costa Rica y Bachiller en Educación Preescolar, también de la Universidad de Costa Rica. Se ha desempeñado como supervisora de Práctica Docente de la carrera de Educación Preescolar de la Universidad de Costa Rica y como profesora de la Sección Preescolar de esta universidad, en cursos como Experiencia Profesional I, Ciencias en la Educación Inicial, Estrategias para el desarrollo de la creatividad del niño (Sede de Guanacaste) y directora de Práctica docente de Graduación para optar al título de Licenciatura en Educación Preescolar. Actualmente labora en el Ministerio de Educación Pública como Docente 
de Educación Preescolar en el Jardín de Niños Porfirio Brenes Castro.

Iván Solano Zúñiga: Es bachillerato con honores en Educación Diversificada del Colegio de San Luis Gonzaga de Cartago. Actualmente es estudiante de tercer año de la Escuela de Ciencias de la Computación e Informática de la Universidad de Costa Rica. Dentro de sus labores se ha proyectado a la comunidad trabajando con jóvenes y en labor social. Fue participante del congreso de universitarios UNIV 2002 organizado por el ICU (Instituto para la Cooperación Universitaria) y realizado en Roma.

Floria Castrillo Brenes: Es Bachiller en Ciencias y Letras, Colegio Nuestra Señora del Sión, estudió Ingeniería Civil, U.C.R., ingresa a la Casa del Artista. Actualmente es Bachiller en Artes Plásticas con énfasis en Grabado de la Universidad de Costa Rica. Ha realizado estudios complementarios tales como: Curso Taller de Papel Hecho a Mano, Prof. Diane Borden Jones Tampa Florida, en U.C.R., Curso Taller de Técnicas Artísticas básicas para Metal, Prof. Loyd Jones, Tampa Florida, en U.C.R., Taller de Grabado en Metal, Maestro Edgar Correal, Museo Rayo, Colombia, Curso Taller de Papel Hecho a Mano, Grabado en Metal y Litografía, Prof. Paul \& Alece Martin - Bufalo University, en U.C.R., Taller de Papel Hecho a Mano para Técnicas de Grabado, Prof. Peter Sowisky - New York, en U.C.R., Curso Libre "Fibras Vegetales para Producción de Papel", Prof. Lorena Blanco, Escuela de Ingeniería U.C.R., Curso "Introducción a la Museología" patrocinado por Museo Nacional, Dirección General del Servicio Civil y U.C.R., Curso "Programación de la Conserva- ción Preventiva en las Instituciones", U.C.R. Ha realizado exposiciones colectivas tales como: Exposición Retrospectiva, Semana Universitaria, Facultad de Artes Musicales U.C.R., "Grabado" Homenaje a Juan Luis Rodríguez, Instituto Tecnológico de Costa Rica, Grabado en Metal, Museo Omar Rayo, Colombia, " Arte Emergente", Sala Brasil, Embajada de Brasil Costa Rica, "Homenaje al Maestro Francisco Amighetti", Centro Costarricense de la Ciencia y La Cultura, Papel Hecho a Mano, "Un Espacio para la Creatividad 96", Facultad de Artes Plásticas U.C.R., "Estampa Joven", Auditorio Manuel Jiménez Borbón, Periódico La Nación, "Grabado y Cerámica" Centro Cultural Costarricense Norteamericano, "Grabado en Madera U.C.R." Exposición Itinerante. algunos reconocimientos son: 1968 Primer Premio, Concurso "Escudos del Liceo Franco Costarricense", 1992 Primer Premio, Afiche IX Seminario y X Congreso Confederación Latinoamericana de Trabajadores las Comunicaciones, 1994 Finalista de la Premiación Concurso "Arte Emergente", Embajada de Brasil, Costa Rica, 1996 Primer Lugar Compartido, Certamen de Artes Visuales "Un Espacio para la Creatividad 96", U.C.R. Tiene una experiencia profesional como Asistente del Taller de Papel Hecho a Mano y su Proyecto de Investigación. Escuela de Artes Plásticas de la U.C.R., Impresora de la Obra del Maestro Pintor y Grabador Francisco Amighetti, Expositora en las "Jornadas de Investigación" Universidad de Costa Rica, Asistente Curso de Producción de Papel Hecho a Mano para fines Artísticos y Artesanales, auspiciado por la O.E.A. y la U.C.R., Asistente en Cursos de Papel Hecho a Mano y Xilografía, auspiciado por Florida Craftsmen Gallery, en St Petersburg, Florida. 
